\title{
Tagungsforum
}

\section{Schwerpunkte im Jurastudium: Zweite Fachtagung des Instituts für Rechtsdidaktik an der Universität Passau}

\author{
Christina-Maria Leeb/Alexander Seidl ${ }^{1}$
}

Am 11. und 12. September 2013 fand an der Universität Passau die vom Institut für Rechtsdidaktik veranstaltete Fachtagung unter dem Titel „Schwerpunkte im Jurastudium “ statt. Rund 80 Teilnehmer verschiedener juristischer Fakultäten aus ganz Deutschland fanden sich zur zweitägigen Tagung ein, die zwölf Fachvorträge zu den Themenblöcken „Schwerpunktbereich“ und „Wissenschaftliches Arbeiten“ sowie eine abschließende Podiumsdiskussion umfasste.

Der erste Konferenztag wurde mit der Begrüßung seitens des Sprechers des Instituts für Rechtsdidaktik, Prof. Dr. Urs Kramer, eröffnet. Kramer stellte in seinen Grußworten die Bedeutung der Rechtsdidaktik in der Hochschullehre heraus und erörterte die Themenauswahl der Tagung. Zum Kanon der in der Hochschuldidaktik diskutierten Konzepte gehöre insbesondere das Modell des juristischen Schwerpunktstudiums. Zehn Jahre Schwerpunktbereiche als Teil der Ersten Juristischen Prüfung seien genug Grund, um Bilanz zu ziehen: Haben sich die Erwartungen erfüllt, die mit deren Einführung verbunden waren? Gibt es Verbesserungsvorschläge? Diesen und ähnlichen Fragen sollte auf der Tagung nachgegangen werden. Dem schlossen sich die Grußworte des Präsidenten der Universität Passau, Prof. Dr. Burkhard Freitag, an, der die Entwicklung des Instituts für Rechtsdidaktik darstellte und diesem eine wichtige Rolle zusprach, weil es die Entwicklungsperspektive der Universität Passau in Lehre und Forschung versinnbildliche. Anschließend betonte der Dekan der Juristischen Fakultät, Prof. Dr. Rainer Wernsmann, dass - nachdem vor zwei Jahren auf diesem Gebiet noch ein hoher Literaturmangel herrschte und auch im Allgemeinen keine große Bewegung in diesem Fachgebiet stattfand - die Rechtsdidaktik immer mehr an Bedeutung gewinne. Nach einigen kritischen Überlegungen zum Schwerpunktstudium an sich leitete Wernsmann auf den ersten Fachvortrag von Ministerialdirigent a. D. Dr. h.c. Heino Schöbel über.

Schöbel, ehemaliger Leiter des Landesjustizprüfungsamtes Bayern, sprach in seinem Vortrag „Das Gesetz zum Schwerpunktbereich - Entstehungsgeschichte und Ziele“ über die Genese und Entwicklung des Gesetzes zur Reform der Juristenausbildung von 2002, ${ }^{2}$ mit dem die Schwerpunktbereiche ins juristische Studium Eingang fanden. Er stellte heraus, dass die Motive für dieses Gesetz die finanzielle und personelle Entlastung der Justiz, die Stärkung der Anwaltschaft und die Reduzierung der Stu-

1 Die Autorin Leeb ist selbstständige Verfahrensbeiständin sowie Ergänzungs- und Verfahrenspflegerin. Der Autor Seidl ist Akad. Rat a. Z. und Doktorand am Lehrstuhl für Öffentliches Recht, Sicherheitsrecht und Internetrecht (Prof. Dr. Heckmann) an der Universität Passau.

2 Gesetz v. 11.7.2002 (BGBl. I S. 2592). 
dierendenzahlen darstellten. Ausweislich der Gesetzesbegründung habe die Reform u.a. die frühzeitige und gezielte Berufsorientierung, schnellere und flexiblere Anpassung der Lehr- und Prüfungsinhalte im Schwerpunktbereich an moderne Entwicklungen, ein breiteres Spektrum an Wahlfächern sowie Profilbildung und Qualitätswettbewerb als Ziele verfolgt.

Im zweiten Fachvortrag hielten Prof. Dr. Christian von Coelln und Dr. Helga Wessel, Universität zu Köln, ein erstes Plädoyer zur Streichung der Schwerpunktbereichsausbildung. Sie stellten die Auswirkungen der Einführung der Schwerpunktbereiche auf die Fakultäten, die Professoren/-innen, die Studierenden und auf Staat und Gesellschaft dar. Besonders kritisierten sie, dass die bisherige hohe Aussagekraft juristischer Noten aufgrund der Noteninflation in den Schwerpunktbereichen immer mehr abnehme und dies zu einer fehlenden Vergleichbarkeit zwischen den Studierenden untereinander und den Universitäten führe. Deshalb sprachen sich die Vortragenden im Ergebnis für eine Abschaffung der Schwerpunktbereiche aus.

Prof. Dr. Christoph Hermann, Universität Passau, referierte über Vor- und Nachteile sowie Reformperspektiven im Schwerpunktbereich. Nachteile seien neben einer Überfrachtung des Lernstoffs und der Vernachlässigung des Pflichtfachstudiums auch die Beeinträchtigung der (Auslands-)Mobilität der Studierenden. Positiv zu bewerten sei jedoch die hohe Identifikation, Motivation und Begeisterung der Studierenden, sofern eine reflektierte Wahl des Schwerpunktbereichs getroffen wurde. Darüber hinaus stelle das Schwerpunktstudium die einzige Chance zur fachlichen Profilbildung für Fakultäten und Studierende gleichermaßen dar. Als Reformmodell schlug Herrmann vor, den Schwerpunkt als weiteres einjähriges Studium nach einem dreieinhalbjährigen Studium im Pflichtfachbereich samt Erster Juristischer Staatsprüfung absolvieren zu lassen. Dieses könnte nach seinen Vorstellungen gegebenenfalls mit einem Mastertitel kombiniert werden und als Voraussetzung für den Eintritt in das Referendariat dienen.

Nach der Kaffeepause informierte der als abgeordneter Praktiker in der universitären Juristenausbildung tätige Richter am Landgericht Dr. Frank Bleckmann, Universität Konstanz, im Rahmen des zweiten Themenblocks über die Förderung wissenschaftlichen Arbeitens in der Studieneingangsphase. Dies verdeutlichte er anhand verschiedener Thesen; u.a. lasse sich das notwendige Orientierungswissen in den gegebenen curricularen- und Lehrorganisationsstrukturen nur in einer Zusatzveranstaltung verlässlich vermitteln. Hier setze das Konzept „Jura lernen!“ der Universität Konstanz an: Neben der Vermittlung von Gesetzes- und Falllösungsstruktur werde den Studierenden dort insbesondere die wissenschaftliche Auseinandersetzung im Recht, wie etwa die Herangehensweise in Klausuren oder die Vorbereitung von Hausarbeiten, näher gebracht.

Der „Förderung der Befähigung zum wissenschaftlichen Arbeiten im Regensburger Schwerpunktbereichskonzept “ war das nächste Fachreferat in diesem Themenmodul gewidmet. Darin stellte Prof. Dr. Martin Löhnig, Universität Regensburg, die Umsetzung des Schwerpunktbereichsstudiums in Regensburg vor. Hierfür wurde eine 
eigene Vorlesung zu Fragen wissenschaftlichen Arbeitens und juristischer Methodik eingeführt; daneben wird vor der eigentlichen Seminararbeit eine Proseminararbeit unter intensiver Begleitung und Korrektur mit anschließendem Bewertungsgespräch angeboten. Die Proseminararbeit ist Zulassungsvoraussetzung zur Studienarbeit, die neben der mündlichen Prüfung die zu erbringende Leistung der juristischen Universitätsprüfung darstellt; eine Klausur findet indes nicht statt.

Als erster Redner des zweiten Konferenztages sprach sich - nach Eröffnung durch Prof. Dr. Tomas Kuhn - Wiss. Mit. Jan Singbartl von der Ludwig-Maximilians-Universität München in seinem Vortrag für die Abschaffung der Schwerpunktbereiche aus. Das Schwerpunktbereichsstudium in seiner jetzigen Konzeption zwinge zu einer zu frühzeitigen Spezialisierung und einer damit einhergehenden inhaltlichen und zeitlichen Überforderung der Studierenden. Obwohl der Vortragende für eine Abschaffung der Schwerpunktbereiche argumentierte, war er sich sicher, dass diese Forderung nicht umgesetzt werde, und plädierte deshalb für eine stärkere Rückkopplung zwischen Lehrenden und Studierenden, eine intensivere Fokussierung auf die Anwaltsausbildung (Vermittlung anwaltlichen Berufsrechts) sowie einen späteren Beginn des Schwerpunktbereichs.

Im Anschluss daran zog Wiss. Mit. Anna Haßfurter, Universität Passau, mit Blick auf den Schwerpunktbereich „Grundlagen des Rechts und des Staates“ eine Zwischenbilanz aus Sicht beteiligter Studierender, Absolventen/-innen und Dozenten/-innen. Problematisch sei aus ihrer Sicht vor allem, dass das Schwerpunktstudium in Passau faktisch drei Semester umfasse. Die Prüfungsordnung sehe vor, dass die mündliche Prüfung frühestens dann abgelegt werden könne, wenn die Ergebnisse von Klausur und Seminar vorliegen. Deshalb erfolge die mündliche Prüfung im Schwerpunktbereich regelmäßig erst im siebten Fachsemester. Haßfurter kam zu dem Schluss, dass eine Modifizierung des bestehenden Schwerpunktbereichskonzepts dahingehend anzustreben sei, dass der Schwerpunkt in nur zwei Semestern abgelegt werden könne und mehr Raum für wissenschaftliche Diskussionen geschaffen werde.

Einen Einblick in die Juristenausbildung in der Schweiz lieferte Rechtsanwalt Dr. Rafael Küffer, Universität Bern, mit seinem Vortrag „Schwerpunktsetzung im Studium - Risiko oder Chance? “. Aufgrund der Bologna-Reform wurde das juristische Studium in der Schweiz auf Bachelor und Master umgestellt, weshalb sich an die universitäre Ausbildung kein Staatsexamen anschließt. Während das Bachelorstudium weitgehend einheitlich verläuft, ist eine Spezialisierung erstmals im universitären Masterstudium möglich, in dem bestimmte Veranstaltungen gewählt werden können. Alternativ zum universitären Schwerpunktmodell kann ein praxisorientierter Schwerpunkt nach der Anwaltsausbildung (Fachanwalt) absolviert werden, der laut Küffer aufgrund der Stellenanforderungen in der Schweiz zu favorisieren ist.

„Notengebung im Schwerpunktbereich - wirklich inflationär?“ lautete der Vortrag von Wiss. Mit. Lukas Beck, Universität Würzburg, in dem er - nach einleitenden Worten durch Prof. Dr. Holm Putzke - die Notengebung in der juristischen Universitätsausbildung an zwei Vergleichsobjekten maß: einerseits am Staatsteil (Binnen- 
vergleich), andererseits an anderen Studiengängen, namentlich Medizin und Ingenieurswissenschaften (Außenvergleich). Einen Reputationsverlust befürchtet Beck nicht, da in anderen Studienfächern noch bessere Ergebnisse erzielt würden, diese an Reputation jedoch seit jeher nichts einbüßten.

Dr. Michael Beurskens, Universität Düsseldorf, referierte zum Thema „Mut zur Lücke - nicht nur im Schwerpunktbereich “. Zu Beginn rechtfertigte er aufgrund von drei aktuellen Symptomen den Titel seines Vortrags. So bestünde mehr als jeder Dritte die staatliche Pflichtfachprüfung nicht; weiterhin seien die Prüfungsanforderungen bundesweit uneinheitlich und selbst auf Landesebene weitgehend unbestimmt. Diese Symptome erklärte er anhand folgender Fakten: Insbesondere hätten sich einerseits die beruflichen Anforderungen dahingehend verändert, dass ein Großteil der Absolventen/-innen den Anwaltsberuf ergreife, und zum anderen sei zu konstatieren, dass die Studierenden kein Studium, sondern eine Ausbildung wollten (lediglich 11 \% hätten während des Studiums Interesse am wissenschaftlichen Arbeiten). Als Fazit präsentierte Beurskens einen aus drei Elementen bestehenden Lösungsansatz: Zunächst sollten die Pflichtfächer einer Radikalkur unterzogen werden; weiterhin sollten die Studierenden - im Gegensatz zu starren Schwerpunktbereichen - die Veranstaltungen frei wählen können. Schließlich sollte das Können, nicht nur das auswendig gelernte Wissen abgeprüft werden. So müssten z.B. Onlinedatenbanken zur Klausurlösung zugelassen werden.

Drei Schritte zur richtigen Methode für das Lehren und Prüfen wissenschaftlicher Arbeitstechniken stellte Akad. Rat Dr. Denis Basak, Universität Frankfurt/Main, vor: Wissenschaftlichkeit, methodisches Setting („Constructive Alignment“) und praktische Auswertung der Studien- und Prüfungsordnungen. Aus Letzterer zog $B a$ sak u.a. die Folgerung, dass freiwillige Angebote zur Methodenlehre zu wenig in den Curricula der Schwerpunktbereiche verankert seien. Das Erlernen dieser Techniken solle daher gezielt gefördert, aber auch abgeprüft werden.

Last but not least präsentierte Wiss. Mit. Nora Rzadkowski, Universität Hamburg, in ihrem Vortrag „Wissenschaftliche Kompetenz als Ziel des Schwerpunktstudiums? “ ihre Lösungsansätze zur Definition des Kompetenzbegriffs in der Wissenschaft. Neben Stärken und Schwächen von Kompetenzentwicklungsmodellen zeigte sie zugleich auch deren Chancen und Risiken auf. Als Stärke wies sie u.a. den normgebietsübergreifenden und international anschlussfähigen Dialog über ein unterschiedliches Wissenschafts- und Forschungsverständnis aus. Ein mögliches Risiko so Rzadkowski - liege darin, dass der Kompetenzbegriff die Tatsache verschleiern könne, dass es Unterschiede zwischen den externen Erwartungen und dem internen Verständnis von wissenschaftlicher Kompetenz gibt.

Den Höhepunkt am Ende des zweiten Veranstaltungstages bildete die hochkarätig besetzte Diskussionsrunde zum Pro und Contra von Schwerpunktbereichen unter Beteiligung von Prof. Dr. Barbara Dauner-Lieb, Universität zu Köln, Richter am BGH Prof. Dr. Henning Radtke, Deutscher Juristen-Fakultätentag, Prof. Dr. Christian Wolf, Universität Hannover, Julia Hörnig, Vorstandsvorsitzende des Bundes- 
verbandes rechtswissenschaftlicher Fachschaften, und Rechtsanwältin Dr. Petra Linsmeier, Kanzlei Gleiss Lutz. Moderiert wurde die Diskussion von Rechtsanwalt Tobias Freudenberg, Schriftleiter der NJW. Dabei wurde insgesamt - interessanterweise - weniger das „Ob“, sondern vielmehr das „Wie“ des universitären Schwerpunktbereichs im Jurastudium kritisiert. So lägen die Ursachen des defizitären Status quo insbesondere in Umsetzungsproblemen, wie etwa einem unzureichenden Verwaltungsapparat im Bereich des Prüfungswesens, oder auch - aus Gleichheitsgesichtspunkten heraus - in fehlenden gesetzgeberischen Vorgaben über die konkrete Ausgestaltung des Schwerpunktstudiums. Während der „richtige“ Zeitpunkt für die Absolvierung des Schwerpunkts kontrovers diskutiert wurde, bestand zwischen den Podiumsteilnehmern weitgehend Einigkeit darin, dass das Schwerpunktstudium an Akzeptanz bei Lehrenden und Lernenden gleichermaßen hinzugewinnen müsse, damit sich der damit verbundene Aufwand lohne. Darüber hinaus solle durch den universitären Teil der Ersten Juristischen Prüfung erreicht werden, dass sich die Studierenden mit einer juristischen Thematik vertieft wissenschaftlich auseinandersetzen. $\mathrm{Ob}$ und wie dies durch eine Optimierung des Schwerpunktstudiums geschehen könne, wurde von den Teilnehmern wiederum teilweise sehr unterschiedlich bewertet.

Prof. Dr. Urs Kramer oblag es, das Resümee über die Veranstaltung zu ziehen und einen Ausblick auf künftige Entwicklungen zu wagen. Er stellte auch in Aussicht, dass im übernächsten Jahr aufgrund des großen Zuspruchs wieder eine solche Fachtagung des Instituts für Rechtsdidaktik an der Universität Passau stattfinden werde.

Auch der Networking-Gedanke kam in den Kaffee- und Mittagspausen und vor allem beim gemeinsamen Abendessen im Altstadt-Hotel am Ende des ersten Konferenztages nicht zu kurz. Für Unterhaltung beim Abendprogramm sorgte ein launiger Vortrag von Ministerialdirigent a. D. Dr. h.c. Heino Schöbel zur „Ausbildungsreform in Absurdistan“.

Insgesamt war die Zweite Fachtagung des Instituts für Rechtsdidaktik eine äußerst gelungene Veranstaltung, die ganz konkrete Verbesserungsvorschläge hinsichtlich der Schwerpunktbereiche bzw. Alternativen hierzu aufzeigte, wobei diese bis zuletzt kontrovers und ergebnisoffen diskutiert wurden. Positiv zu bewerten ist ferner, dass an verschiedenen Stellen auch die Sichtweise der Studierenden in die Diskussion miteinbezogen wurde. Wünschenswert wäre insoweit jedoch noch eine umfassendere Befragung, um auch die Meinung derjenigen, die von der Thematik der Tagung unmittelbar betroffen sind, bei künftigen Entscheidungen zu diesem Themenkomplex stärker zu berücksichtigen. 